\title{
Estrategias motivacionales efectivas en profesores en formación ${ }^{1}$
}

\author{
Jorge VALENZUELA² \\ Carla MUÑOZ ${ }^{3}$ \\ Abigail MONTOYA LEVINAO ${ }^{4}$
}

\section{Resumen}

Este artículo presenta los resultados de una investigación realizada en Chile, cuyo objetivo fue, en primer lugar, testear la hipótesis de que profesores en formación de último año tienden, mayoritariamente, a concebir las estrategias motivacionales efectivas desde una lógica de entretención, por sobre otras vinculadas más directamente con la realización de la tarea o la motivación por aprendizaje; y en segundo lugar, caracterizar las estrategias motivacionales percibidas como posibles y efectivas, agrupándolas según su foco en estrategias orientadas al aprendizaje, realización de la tarea y a la entretención. En el estudio participaron 306 estudiantes de pedagogía chilenos, de ambos sexos, de distintas universidades de la Región de la Araucanía que cursan su último año de carrera. Los resultados muestran la confirmación de la hipótesis, evidenciando una preeminencia de una concepción de la motivación escolar que se organiza en torno a la idea de entretención. Más aún, se evidencia que esta lógica de la entretención invisibiliza, en muchos casos, una concepción de la motivación escolar centrada en favorecer el deseo de aprender. Finalmente, se discuten las implicancias de estos hallazgos para una mejor formación docente y para un ejercicio efectivo de la motivación en el aula, en donde se tomen en consideración los aspectos relacionados con la entretención y la realización de la tarea, pero donde prevalezca la motivación orientada a suscitar el deseo de aprender.

\section{Palabras clave}

Motivación escolar - Estrategias motivacionales - Estudiantes de pedagogía - Efectividad - Representaciones.

\footnotetext{
1- Esta investigación ha sido financiada por la Comisión Nacional de Investigación en Ciencia y Tecnología (CONICYT - Chile) a través del proyecto Fondecyt Regular 1150533.

2- Universidad de Playa Ancha, Valparaíso, Chile. Contacto: valenzu@gmail.com

3- Pontificia Universidad Católica de Valparaíso, Valparaíso, Chile. Contacto: carla.munoz@pucv.cl

4- Universidad Católica de Temuco, Temuco, Chile. Contacto: rayen22quitral@gmail.com
} 


\section{Effective motivational strategies in pre-service teachers}

\section{Abstract}

This article presents results from a study conducted in Chile whose objective was to test the hypothesis that most pre-service teachers in their last year's training tend mainly to conceive the effective motivational strategies based on the logic of amusement rather than from others more directly related to accomplishing the task or the motivation for learning. Secondly, to characterize the perceived motivational strategies as possible and effective, grouping them according to their focus on learning-oriented strategies, accomplishing the task, and amusement. The study involved 306 male/female students of Chilean pedagogy, from different universities in the Region of Araucania who are in their final year of professional studies. The results confirm hypothesis showing the pre-eminence of a conception of school motivation organized around the idea of entertainment. Moreover, it shows that this logic of amusement, in many cases, makes invisible a conception of school motivation focused on behalf of the desire to learn. Finally, we discuss the implications of these findings to improve teacher training and to make motivation effective in the classroom where the aspects related to the amusement and accomplishing the task are taken into account, but where the motivation to arouse the desire to learn prevails.

\section{Keywords}

School motivation - Motivational strategies - Pre-service teachers - Effectiveness Representations.

\section{Introducción}

La motivación escolar es un tema de creciente interés en el área educacional y a nivel investigativo es reconocida como una variable clave en el aprendizaje (ROBBINS et al., 2004; HATTIE, 2009). Por su parte, la motivación escolar ha sido reconocida como una dimensión importante en la enseñanza por la Política Pública en educación (CHILE, 2003); y más recientemente, como uno de los indicadores del Sistema de Medición de la Calidad en Educación, SIMCE (CHILE, 2014a, 2014b). Desafortunadamente, si bien este reconocimiento general es una señal positiva, no basta. Se requiere ir un poco más lejos, por ejemplo, definiendo con más claridad qué se entiende por motivación y cómo esta se operacionaliza.

Como explicamos más adelante, la motivación no es un constructo unitario. $\mathrm{Su}$ concepción adopta distintas formas dependiendo del objeto específico de ésta (GOLLWITZER; OETTINGEN, 2001). Así, la forma de concebir la motivación condiciona el tipo de dispositivos motivacionales posibles de implementar. 
Este estudio indaga sobre los tipos de estrategias que los futuros profesores visualizan como posibles y más efectivos para incrementar la motivación en contexto escolar. Se entiende que estas estrategias son comprendidas por el profesor a partir de las representaciones que estos tienen acerca de este constructo y de las concepciones que subyacen a éste, constituyendo el marco para las posibles acciones y estrategias motivacionales.

En concreto, el objetivo de esta investigación se centra en testear la hipótesis de que estos profesores en formación visualizan como posibles y efectivas, estrategias que se estructuran a partir de la lógica de que motivar es entretener, por sobre otras lógicas en las cuales motivar es suscitar el deseo de realizar la actividad o suscitar el deseo y la activación de recursos cognitivos para aprender. Y junto con lo anterior, caracterizar las estrategias agrupándolas en estas tres representaciones o lógicas de comprensión de la motivación escolar.

\section{Representaciones acerca de la motivación escolar}

Existen diversas formas de representarse la motivación escolar. Estas distintas formas, de alguna manera, se constituyen en el marco desde el cual adquieren sentido y son posibles un cierto tipo de acciones y dispositivos pedagógicos al interior del aula.

Un estudio reciente de Valenzuela y otros autores (2014) distinguió tres focos articuladores de las representaciones de la Motivación escolar. Esta clasificación surge del análisis de grupos focales con profesores en formación y en ejercicio y dan lugar a tres tipos de concepciones distintas sobre la motivación escolar: a) la motivación escolar que se centra en el querer aprender los contenidos de la clase, b) la motivación escolar entendida como el deseo y compromiso de realizar la tarea propuesta por el docente y finalmente, c) la motivación escolar como el entusiasmo que manifiesta el alumno durante la clase.

De esta manera y entendiendo que las estrategias son métodos, técnicas y/o procedimientos que tienen por objetivo alcanzar una meta y que regulan el actuar de la persona en cuestión (MALLART NAVARRA, 2000), queda claro que su diseño puede estar influido por las representaciones que tienen los docentes acerca del ámbito específico de aplicación, en este caso, de la motivación escolar.

\section{Tres focos en la representación de la motivación}

\section{a) Motivación hacia el aprendizaje}

Motivar hacia el aprendizaje significa estimular la voluntad de indagar para la construcción de conocimientos y el desarrollo de habilidades (HUERTAS, 1997; VIAU, 2009). Lo anterior implica activar recursos cognitivos para dominar un conocimiento, transformarlo y utilizarlo en otras realidades, estableciendo relaciones de carácter significativo entre los conocimientos previos que se poseen y la información proporcionada en el contexto de aprendizaje, de manera no arbitraria y sustantiva (AUSUBEL; NOVACK; HENESIAN, 1989; MOREIRA, 2012). Así, las estrategias de motivación con foco en el aprendizaje son aquellas dirigidas a que el alumno quiera aprender de manera profunda, desarrollando sus habilidades y enriqueciendo sus conocimientos, mediante la vinculación efectiva de las distintas disciplinas y sus realidades inmediatas. 
Pese a que la motivación es un proceso interno al sujeto, está sujeta a las relaciones interpersonales dentro del aula (ALONSO TAPIA, 2009), es decir, el docente como agente motivador extrínseco puede contribuir significativamente a estimular el deseo de aprender de manera profunda en cada individuo (THOONEN et al., 2011).

A pesar de que pareciera obvio que el esfuerzo de los docentes se dirige, finalmente, hacia el aprendizaje, la evidencia muestra que muchas veces se yerra el camino y el dispositivo que se implementa sólo alcanza para motivar puntualmente por la tarea o para crear las condiciones de un buen clima de aprendizaje, quedando en deuda el suscitar en el estudiante la necesidad y el deseo profundo de aprender aquello que la escuela intenciona (PRECHT et al., 2016).

\section{b) Motivación como realización de la tarea}

La motivación entendida como realización de la tarea es aquella que refiere principalmente al logro de las actividades encomendadas por el docente como labor especifica realizada en un tiempo determinado, plano en el cual la literatura especializada centra la mayor parte de sus contribuciones (ELLIOT et al., 2005; GARCÍA LÓPEZ, 2008; ALDERMAN, 2013). Desde esta perspectiva, las estrategias motivacionales apuntan a que el estudiante desarrolle de la mejor manera posible las actividades propuestas y cuyo nivel de logro muchas veces es fundamental para establecer parámetros de rendimiento escolar.

El grado de motivación que el estudiante tenga respecto del logro de la tarea va a estar condicionado por factores como la importancia, el interés y la utilidad de ésta (ECCLES, 2005; WIGFIELD; TONKS; KLAUDA, 2009; WIGFIELD; CAMBRIA, 2010), el costo que se está dispuesto a asumir (ANDERSON, 2000; NEUVILLE; BOURGEOIS; FRENAY, 2004; BARRON; HULLEMAN, 2015; FLAKE et al., 2015) y del sentimiento de competencia que tenga hacia la misma (BANDURA; RAMACHAUDRAN, 1994; BONG, 2001; WIGFIELD; TONKS; KLAUDA, 2009). Cuando el foco está puesto en la tarea son éstos los factores que direccionan la acción motivacional del docente, cuyo interés es que los estudiantes realicen las actividades que ha planificado.

La concreción de la tarea, por su parte, permite al docente constatar aprendizajes. Sin embargo, cuando el interés sólo se centra en el logro de ésta como fin último (meta de desempeño), el aprendizaje pasa a un segundo plano y la motivación del estudiante tiende a focalizarse en la recompensa de la tarea lograda o en evitar una sanción (AMES, 1992; PINTRICH, 2000; BANDURA; LOCKE, 2003; ELLIOT et al., 2005).

\section{c) Motivación como entretención}

Una tercera manera de concebir la motivación escolar es en clave de entretención (KIM; KIM, 2016), es decir, como la generación de un espacio de agrado, orden y seguridad que disponga (o no indisponga) al estudiante frente a las tareas que mediarán su aprendizaje (MARZANO et al., 2005). En este contexto, el clima de aula se vuelve fundamental (LÓPEZ; BILBA0; RODRÍGUEZ, 2012; MORA; CRUZ; SOUSA, 2013; SIMÓN; GÓMEZ; ALONSO-TAPIA, 2013). No obstante, este clima de aula que se debiera estructurar 
a partir de las cuatro dimensiones: física, funcional, funcional temporal y relacional (IGLESIA, 2008), normalmente está orientado a satisfacer necesidades de comodidad y orden en los estudiantes (MARZANO et al., 2005), para que se predispongan a hacer lo que la escuela les propone. Muchas veces esta preocupación por el clima de aula se convierte en un fin en sí mismo y no en una condición inicial y necesaria para generar un interés en el estudiante por aprender (JUVONEN; WENTZEL, 1996; HUERTAS, 1997). En esta perspectiva, el docente busca mantener un buen clima de aula, en el que el niño se sienta cómodo, seguro, aceptado, entretenido y comprendido, como base para poder pasar la materia (PRECHT et al., 2016), desplazando eventualmente a un segundo plano el que estos esfuerzos motivacionales puedan apuntar directamente a que el estudiante quiera aprender (SILVA-PEÑA; NAVARRETE; ZUÑIGA, 2012).

Ahora bien, si preguntásemos a los profesores cuál es el foco más importante para la motivación escolar, muy probablemente aparecería nítidamente que ésta debe orientarse al aprendizaje. No obstante, esta declaración no siempre se condice con la realidad y muchas veces en la práctica, los esfuerzos motivacionales están orientados al foco de entretención, entendiendo que el espacio educativo a nivel de aula es fundamental y condición basal para el desarrollo de la clase (PRECHT et al., 2016).

El problema se presenta cuando nos quedamos en ese nivel, sin comprender que hacer la clase no es lo mismo que lograr que los estudiantes aprendan o que el deseo de aprender no se genera automáticamente en un contexto entretenido. El aprendizaje requiere de varios factores, y entre ellos, quizá el más fundamental, es que aprender en contexto escolar debe tener sentido para el estudiante.

En este contexto, la representación que se tiene del objeto (en este caso de la motivación escolar) condiciona de alguna manera la actividad del profesor. Si se concibe la motivación escolar como entretener, eventualmente se logrará una actividad entretenida, pero no necesariamente el deseo de los estudiantes de apropiarse de los contenidos o habilidades propuestos para su aprendizaje.

Por lo anterior, el objetivo de este estudio es poder analizar las estrategias y dispositivos motivacionales que los profesores visualizan como posibles y como más efectivos para incrementar la motivación escolar y, a partir de ellos, testear la hipótesis de que esta última forma de concebir la motivación es predominante en estudiantes que están a punto de ingresar al sistema escolar como profesores. Asimismo, testear la hipótesis de que la motivación entendida como suscitar el deseo de aprender ocupa un rol secundario a la hora de visualizar estrategias para mejorar la motivación escolar.

\section{Método}

\section{Participantes}

La muestra de este estudio estuvo constituida por estudiantes de pedagogía $(n=306)$ de ambos sexos (mujeres $=61 \%$, hombres $=39 \%$ ), con una edad media de 23,7 años $(s d=5,02)$, de universidades públicas (1) y privadas (2) de la zona sur de Chile (Región de la Araucanía), quienes se encontraban cursando los últimos años de sus carreras ( $4^{\circ}$ y $5^{\circ}$ años). Cabe 
consignar que la formación de profesores en Chile se realiza en las universidades y tiene una duración que varía entre 4,5 y 5 años de formación (para una revisión del sistema de formación chileno, ver Contreras-Sanzana y Villalobos-Clavería, 2010).

Es importante precisar, asimismo, que todos los participantes han sido formados en un modelo formativo que considera la inmersión temprana en situaciones prácticas. En este modelo, presente en la gran mayoría de programas de formación de profesores chilenos, las prácticas tempranas corresponden a experiencias que los alumnos tienen de acercamiento a la realidad escolar en tanto docentes. Así, ya desde el primer año los estudiantes realizan estancias en el medio escolar en tanto futuros profesores, realizando actividades de observación participante y colaboraciones menores que le permitan, por una parte, conocer la realidad escolar y por otra, apropiarse paulatinamente del rol docente (GORICHON et al., 2015). Por lo anterior, para los participantes de este estudio la realidad escolar y la dinámica de clase no son desconocidas.

\section{Instrumento}

Para indagar en torno a las estrategias motivacionales que son percibidas por los estudiantes como posibles para promover la motivación escolar y la jerarquía de éstas en función de su eficacia en el aula, se preguntó directamente a los encuestados ¿Qué estrategias se pueden utilizar para generar motivación escolar, y cuáles son las más efectivas? Además de la pregunta por las estrategias, se daban instrucciones para que, junto con anotar las estrategias posibles, éstas fuesen jerarquizadas en función de su grado de efectividad. Estas preguntas fueron piloteadas en una pequeña muestra y posteriormente sometidas a la muestra definitiva. Así, las estrategias referidas por los participantes fueron agrupadas a partir de la jerarquización que hicieron de su efectividad. De esta manera, un primer conjunto de estrategias agrupó aquellas consideradas por la persona que responde como la más eficaz. Un segundo, con aquellas que ocuparon el segundo nivel de jerarquía y un tercer conjunto de estrategias con aquellas, que siendo consideradas efectivas, eran percibidas en un tercer nivel de efectividad.

Estas preguntas formaban parte de una batería más amplia que indagaba sobre variables motivacionales relacionadas con la profesión docente, tales como autoeficacia motivacional (VALENZUELA et al., 2015) y motivos que dan sentido al aprendizaje escolar (VALENZUELA, 2009).

\section{Procedimientos}

Para la aplicación del instrumento, se estableció contacto previo con las autoridades pertinentes de las carreras de pedagogía de las distintas casas de estudio, solicitando la autorización correspondiente. La toma de datos fue efectuada por uno de los investigadores responsables y se realizó de manera colectiva y presencial en sus respectivas instituciones.

Como es de rigor en este tipo de investigaciones, los participantes fueron debidamente informados de sus derechos, luego de lo cual quienes aceptaron participar, firmaron los consentimientos y procedieron a contestar el instrumento. La aplicación de la batería completa no sobrepasó los 30 minutos. 


\section{Procedimientos analíticos}

Los datos de la encuesta fueron transcritos y ordenados en una base de datos, asignando un número a cada participante a manera de resguardar su identidad. Las estrategias que la muestra explicitó fueron categorizadas en tres grandes grupos de acuerdo a su contenido temático, a saber: a) Motivación como aprendizaje, b) Motivación como realización de la tarea y c) Motivación como entretención. Se incluyó también la categoría NO estrategia considerando, en ésta, aquellas respuestas que no responden a la pregunta. Esta clasificación fue realizada por tres evaluadores independientes. Tras la triple categorización se realizó un análisis de los casos en los cuales no hubo acuerdo inter-corrector o había dudas sobre la categorización de la estrategia propuesta por el o la estudiante.

Por su parte, para caracterizar en detalle el contenido específico de las estrategias categorizadas dentro de cada foco, se procedió a un análisis de contenido (GIBBS, 2012) a partir del cual se relevaron las características específicas de las estrategias al interior de cada uno de estos 3 grupos.

\section{Resultados}

\section{Estrategias motivacionales: tipos y percepción de efectividad}

\section{Tipos estrategias}

Como señalamos con anterioridad, esta investigación se centró en indagar las concepciones que subyacen a las estrategias declaradas por los docentes en formación como efectivas para generar Motivación Escolar. De acuerdo con las respuestas entregadas, se establecieron tres categorias: a) estrategias de motivación con foco en el aprendizaje, b) en la tarea y c) en la entretención (VALENZUELA et al., 2014).

Bajo este criterio, los resultados muestran que de un total de 306 casos, hay 698 estrategias válidas (Figuras 1 y 2). De ellas, un 20,49\% corresponden a estrategias que apuntan a promover el deseo de aprender; un 12,18\% están orientadas a que los estudiantes quieran realizar las asignaciones dadas por el profesor y finalmente un 67,34\%, es decir dos terceras partes del total, se orientan a la entretención es decir a mantener la atención de los estudiantes, a evitar que se aburran, a generar un ambiente propicio para la realización de la clase.

Esta distribución (ver Figura 2) mantiene el mismo perfil cuando se desagrega por el grado de efectividad de las estrategias percibido. En todos los casos, las estrategias focalizadas en la entretención, es decir aquellas en las cuales subyace una concepción de motivación vinculada al generar condiciones de agrado para poder realizar la tarea instruccional, (ver Figura 3) son claramente mayoritarias frente a los otros dos tipos de estrategias. Llama la atención asimismo que las estrategias focalizadas en el aprendizaje sólo alcancen el 20\% de las menciones en estos profesores. 
Figura 1: Estrategias para favorecer la motivación escolar: distribución por foco prioritario (en \%).

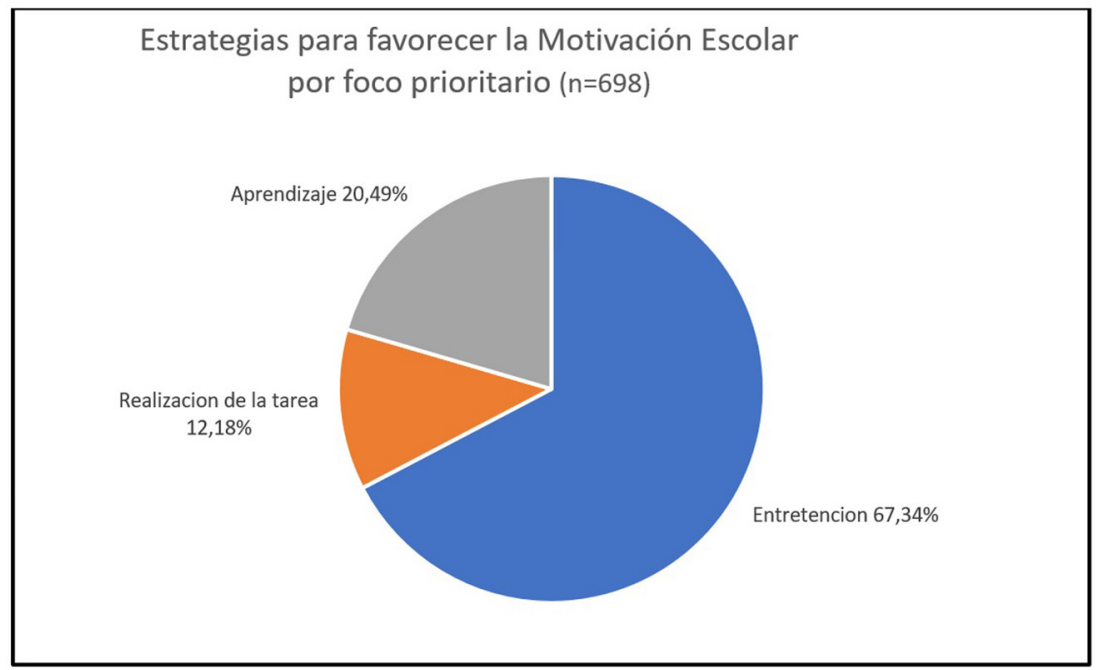

Fuente: Elaboración propia.

\section{Las estrategias más efectivas}

Por su parte, dentro de aquellas estrategias que fueron clasificadas por los participantes como las estrategias más efectivas (nivel de jerarquía 1), se constatan resultados similares: es decir una mayor frecuencia de estrategias que apuntan a la entretención $(67,34 \%)$ seguido de estrategias con foco en el aprendizaje $(20,49 \%)$ y finalmente, aquellas con foco en la Tarea $(12,18 \%)$.

Figura 2- Estrategias motivacionales por foco y jerarquía de efectividad (en \% por cada nivel de efectividad).

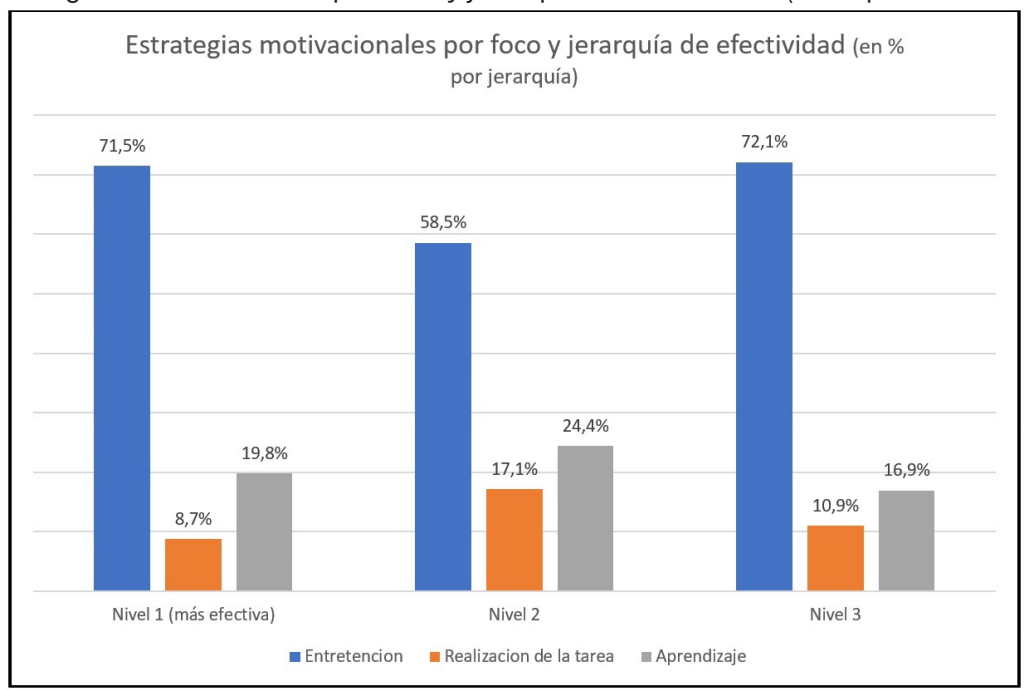

Fuente: Elaboración propia. 
Finalmente, se constata que casi la mitad de los participantes de la muestra (48,69\%) no menciona ninguna estrategia que apunte directamente al aprendizaje.

\section{Focos prevalentes}

Al analizar el conjunto de estrategias referidas por un mismo sujeto, advertimos que en muchos casos existe una tendencia prevalente hacia algunos de estos tres focos. La prevalencia en este caso fue operacionalizada como la presencia mayoritaria de un tipo de estrategia.

En el caso de las estrategias focalizadas en la entretención, se observa que de los 306 casos, una submuestra de 172 sujetos, es decir un 56,2\%, menciona estrategias motivacionales con una tendencia claramente prevalente hacia este foco. Caen dentro de esta categoría aquellos sujetos que: a) sólo marcaron estrategias con foco en la entretención; b) aquellos con dos estrategias Entretención y una estrategia focalizada en la Tarea; y c) aquellos con dos estrategias Entretención y una estrategia focalizada en el Aprendizaje.

Por su parte, la submuestra cuyo foco prioritario y claramente prevalente es la Tarea es decir, donde todas las estrategias son mayoritariamente orientadas hacia este foco, quedó constituida sólo por 11 sujetos (3,6\% de la muestra).

Finalmente, el grupo de personas cuyas estrategias y cuyo foco prevalente fueron estrategias focalizadas en el Aprendizaje estuvo constituida por 24 estudiantes (7,8\%) de los cuales sólo 4 sujetos mencionaron sólo estrategias focalizadas en el aprendizaje, mientras que en los otros 20 hay 2 de 3 estrategias focalizadas en el aprendizaje.

En el resto de la muestra ( $n=99,32,4 \%)$, las estrategias apuntan a diferentes focos, sin que alguna de ellas tenga una especial prevalencia.

Figura 3- Foco prevalente de estrategias motivacionales (en \%).

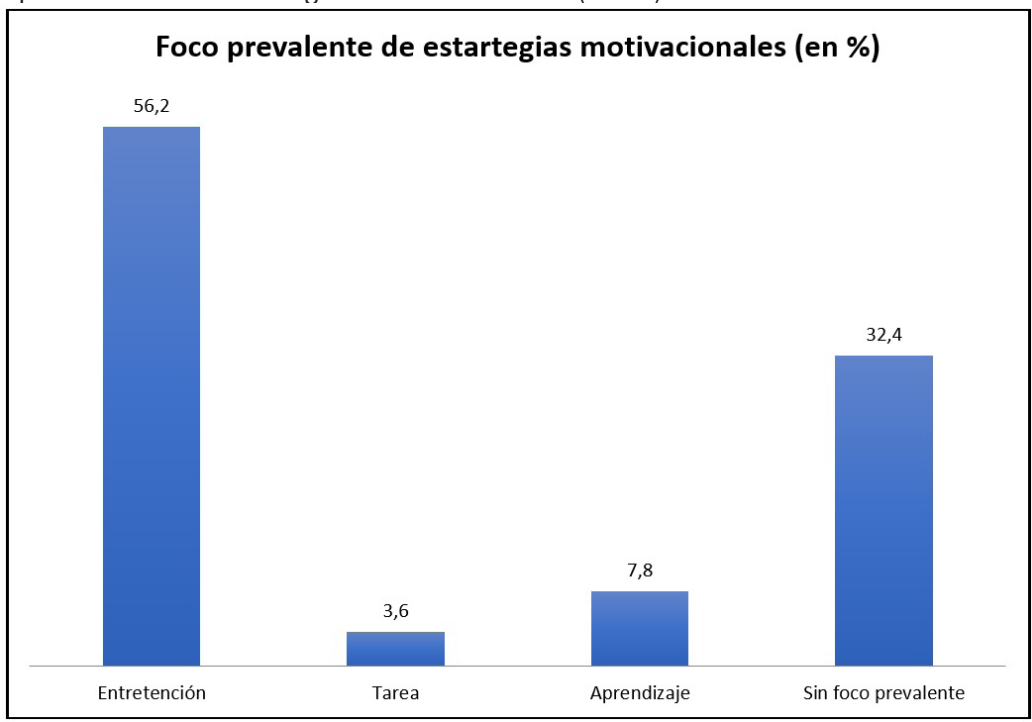

Fuente: Elaboración propia. 
En síntesis, se observa una marcada prevalencia de estrategias motivacionales orientadas a generar las condiciones de agrado y confort que permitan al estudiante entretenerse, y a través de este estado de ánimo, aprovechar de mejor manera la clase, pasando a un segundo plano las dimensiones directamente vinculadas a la realización de la tarea, y lo más preocupante, la del aprendizaje.

\section{Estrategias motivacionales: focos y contenidos específicos}

El primer paso fue analizar el contenido específico de las estrategias que fueron clasificadas a partir de su orientación o foco, es decir: estrategias que se orientan o focalizan en el aprendizaje, en la tarea y en la entretención (VALENZUELA et al., 2014). Se excluyeron del análisis aquellas respuestas que no correspondían a estrategias.

\section{Estrategias motivacionales orientadas hacia el aprendizaje}

Las estrategias de motivación escolar declaradas en este estudio y que son entendidas en clave de aprendizaje apuntan a mostrarle al estudiante la utilidad en la vida diaria de los conocimientos académicos, así como los beneficios que le otorgará el aprendizaje escolar en el futuro, tanto a nivel personal como profesional, y esto, para alcanzar mejor calidad de vida. En esta perspectiva, las estrategias vinculadas al aprendizaje, es decir, orientadas a activar los recursos cognitivos para querer aprender, se articularían en torno al incentivo docente para que los estudiantes busquen querer aprender para sí mismos como motivación intrínseca (BORUCHOVITCH; BZUNECK, 2000; RYAN; DECI, 2017) mediado por la significación de los aprendizajes a través de la vinculación de los conocimientos previos con el nuevo material, comprendido desde la perspectiva teórica de Ausubel, Novack y Henesian (1989). Esta significatividad está mediada por una adecuada contextualización de los contenidos a la realidad del estudiante. De esta manera, actividades desafiantes, actividades en terreno y trabajo con la comunidad y redes de apoyo, surgen como un soporte para alcanzar aprendizajes que les sean significativos en su cotidianidad. En esta perspectiva, se concibe que el aprendizaje en la escuela debe ser útil, tener sentido para el estudiante, debe estar relacionado con su entorno, y así será posible que el estudiante se motive a querer aprender y movilice sus recursos y saberes para ello. En este sentido el profesor puede "[...] demostrar la relevancia de la asignatura con la vida actual y futura del estudiante (mostrar consecuencias positivas)" (Caso 70, E15) o "[...] realizar trabajo colaborativo entre la comunidad educativa, donde los aprendizajes obtenidos sean funcionales, es decir, que los conocimientos puedan ser aplicados a la vida diaria y no sean aprendizajes mecánicos" (Caso 128, E3).

Las estrategias declaradas por los estudiantes con foco en el aprendizaje se caracterizan por considerar la actividad escolar como desafiante cognitivamente, ya sea de manera individual o grupal, abarcando la experimentación, la búsqueda y construcción del conocimiento. Se insiste además, muy probablemente influido por la perspectiva

5- Indica el número de caso y el número jerarquizado de la estrategia (E) mencionada, donde 1 es la estrategia percibida como más efectiva. 
ausubeliana, en que esta estrategia considere los conocimientos previos de los estudiantes y el respeto del ritmo de cada uno de los alumnos como clave de su efectividad ya que las estrategias de motivación escolar más efectivas son aquellas que “[...] están vinculadas a la realidad de cada uno de los estudiantes" y es por ello que es "[...] fundamental que cada docente conozca el contexto del niño y niña, ya que de esta manera generará la motivación a los niños/as adecuada a las características, sociales, culturales, biológicas, sociológicas, etc.”(Caso 26, E1).

\section{Estrategias motivacionales orientadas hacia la realización de la tarea}

En estas estrategias con foco en la tarea, subyace una representación que asocia la motivación escolar directamente con acciones concretas propuestas por el docente y que tendrían una consecuencia positiva inmediata para el estudiante. Dicho de otra manera, son fundamentalmente estrategias motivacionales basadas en el refuerzo externo a través de premios como incentivo, cuyo objeto final es la realización de tareas concretas durante la clase.

Los docentes perciben como eficaces dentro de esta lógica, estrategias que van desde la identificación de los estímulos que serían "llamativos" para el alumno (Caso 298, E1) hasta el "Condicionamiento operante: ya que si el docente ofrece algo a cambio de que estudien, los estudiantes se interesarán por realizar lo solicitado por el profesor" (Caso 166, E3), pasando por una variedad de recompensas y premios para incentivar por meta cumplida, tales como felicitaciones y reconocimientos concretos.

En esta perspectiva, el estudiante estaría motivado a terminar las actividades pedagógicas propuestas por el docente, incentivado por un estímulo. No obstante, desconocemos si hay conciencia de que el hecho de finalizar las actividades propuestas no conlleva necesariamente el aprendizaje efectivo de los saberes intencionados por el docente, aun cuando toda actividad debiese, en teoría, tener este fin último.

Como contrapartida al estímulo, aparece la noción de castigo, que si bien es mencionado una sola vez -Caso 174, E2-, podría tener una mayor prevalencia que la reportada en este estudio, dado que la dinámica "refuerzo y castigo", en tanto resabio del conductismo, es mal visto desde la pedagogía. En cualquier caso, no tenemos suficiente información sobre cuán presente está la noción de castigo dentro de las representaciones de la motivación escolar.

\section{Estrategias orientadas hacia la entretención}

En este foco emergen, a lo menos, tres ejes que articulan las representaciones acerca de la motivación escolar, la cual es vista como acciones que llevan a la recreación y entretención de los estudiantes.

El primer eje de esta representación es aquella que se centra en el diseño de actividades lúdicas y dinámicas como soporte para lograr motivación. En esta clave, utilizan recursos como juegos, música, canciones, material concreto, que sean de interés para presentar el contenido, asegurando así la atención del estudiante y su participación, evitando así el aburrimiento. 
En este sentido, aparece recurrentemente el recurso a las TIC's como mediaciones que pueden aportar a la motivación de los estudiantes, como por ejemplo "Realizar actividades didácticas, es decir, mediante power point, prezi, con el fin de que los estudiantes participen de manera más activa" (Caso 33, E2). En este sentido, llama la atención que, pese a que no se solicitaba, hubo advertencias sobre "[no] abusar de las TIC's" (Caso 219, E3).

Un segundo eje en esta representación relaciona la motivación escolar directamente con el ambiente del aula, y específicamente con el clima emocional y afectivo relacional (con el docente) del estudiante para poder trabajar en clases. En esta línea, es importante que el estudiante se sienta cómodo, seguro, comprendido y en confianza con el docente y sus pares. Esta estrategia requiere del profesor "Ser más comprensivos, ponerse verdaderamente en el lugar de los estudiantes, escucharlos, ponerles atención. La idea es que se sientan importantes para cualquier profesor" (Caso 34, E1). Pero también, un trabajo personal del docente que permita "Formarme como profesor una visión positiva de mis estudiantes, es decir, creer en sus capacidades y darles a conocer esta visión, para que sientan más cercano al docente" (Caso 51, E3).Un tercer eje en esta representación de la motivación escolar, entendida en clave de entretención, considera los intereses, necesidades, gustos personales, características individuales, conocimientos previos y experiencias de los estudiantes como factores que ayudan a que el estudiante se motive. Desde esta perspectiva, el docente debe siempre considerar e incorporar los intereses de los estudiantes en la realización de sus clases.

Esto podría entenderse al menos de dos formas: una de carácter genuino, otra de carácter aparente. Desde una perspectiva genuina, el profesor orienta su acción hacia el aprendizaje, con el fin de "[...] informarse de los gustos de cada uno de los alumnos y hacer de ellos una motivación para cada uno de ellos” (Caso 274, E3) y considerar los “[...] conocimientos previos, experiencias de vida, pero especialmente en abarcar los intereses y gustos de los estudiantes" (Caso 128, E1). La segunda forma que hemos llamado "aparente", supone un interés del docente por conocer los gustos de los estudiantes, para disponerlo al estudiante al trabajo escolar y al aprendizaje, no obstante éste pareciera focalizarse en el entretenimiento como un fin en sí mismo, donde "[...] primero que todo, saber qué es lo que el niño quiere realizar, qué es lo que le gusta hacer..." (Caso 91, E1) ya que una estrategia de motivación "[...] se debe basar en los intereses de los niños, por ende, si a ellos les gustan los juegos, realizar algún tipo de juego, o si le gusta cantar, llevar por ende, si a ellos les gustan los juegos, realizar algún tipo de juego, o si le gusta cantar, llevar algún instrumento y cantar de manera conjunta canciones que sean de su interés" (Caso 13, E1). Así, en este caso, se entretiene al niño para complacerlo sin la intención, explícita al menos, de promover su deseo de aprender.

\section{Discusión y conclusiones}

Existe más de una forma de conceptualizar la Motivación Escolar. En este estudio hemos observado y caracterizado tres maneras de concebirla a partir de las estrategias que los profesores consideran efectivas para promover la motivación escolar. Del total de estrategias motivacionales mencionadas, casi dos tercios responden a una lógica 
articulada por una representación de la motivación escolar centrada en la entretención. De igual manera, al analizar por participantes, un grupo mayoritario de profesores en formación manifiestan una tendencia prevalente a entender la motivación como acciones diseñadas para que el estudiante se sienta entretenido y confortable en la clase, y a partir de ese estado de confort poder captar su atención y/o poder tener un ambiente propicio para realizar la clase (PRECHT et al., 2016). En ese contexto, las dinámicas de carácter lúdico o con apariencia de lúdico (PREISS, 2009), son un recurso novedoso para captar la atención de los estudiantes y generar un clima de agrado. Un segundo grupo, muy minoritario, concibe prevalentemente la motivación como la disposición para realizar las tareas encomendadas por el profesor en la clase, y para favorecerla se recurre a premios e incentivos, en clave de motivación extrínseca (CERASOLI; NICKLIN; FORD, 2014). Finalmente, un tercer grupo de docentes en formación, minoritario también, visualiza la motivación focalizada en el aprendizaje. En este caso, los futuros profesores proponen desafiar a los estudiantes para aprender y comprenden que el estudiante construye su aprendizaje y que ellos tienen un rol mediador que da sentido a lo que está aprendiendo.

Estos resultados podrían entenderse como falta de disponibilidad de estrategias que apunten directamente a suscitar un deseo de aprendizaje. Este tipo de estrategias serían más complejas que el recurso necesario para simplemente captar la atención o entretener. No obstante, como mostramos en los resultados, muchas de las estrategias orientadas al aprendizaje remiten de alguna manera a la idea de aprendizaje significativo de Ausubel (AUSUBEL; NOVACK; HENESIAN, 1989; MOREIRA, 2012), sin poner en cuestión el hecho de que esta perspectiva teórica sólo da cuenta de un aprendizaje por recepción. En esta perspectiva, se entiende que exista la creencia de que para que exista aprendizaje bastaría conectar de manera sustantiva y no arbitraria los conocimientos previos con la materia que se intenta enseñar (AUSUBEL; NOVACK; HENESIAN, 1989). No obstante, si bien esto puede ser un primer paso, no garantiza que estudiante desee aprender, por lo que hay que considerar otros elementos (VALENZUELA, 2007; BORUCHOVITCH; BZUNECK; RUFINI, 2010).

En este contexto, ya sea por lo complejo de su implementación o porque prevalece una representación más cercana a la conceptualización cotidiana de la motivación, hubiésemos esperado que los futuros profesores de la muestra hubiesen relevado con más claridad la orientación hacia el aprendizaje, algo que suponemos consustancial a la profesión.

Más aún, se pudo constatar que un 56\% de los futuros docentes sólo visualiza estrategias motivacionales con foco en la entretención. Es decir, uno de cada dos de ellos cuando concibe una estrategia motivacional para sus estudiantes, lo hace en clave de entretención y que dos terceras partes de todas las estrategias que son visualizadas como posibles, responden a este foco. Y más preocupante aún: que casi la mitad de la muestra no mencionara ninguna estrategia directamente relacionada con suscitar en el estudiante el deseo de aprender. Este alto grado de encapsulamiento de la representación en torno a este eje es consistente con otras investigaciones recientes acerca de la motivación escolar y las representaciones que los docentes en ejercicio tienen sobre esta dimensión del aprendizaje (VALENZUELA et al., 2014).

La pregunta que surge a partir de esta evidencia es ¿Por qué estos docentes tienen la visión de que la entretención es el medio prioritario a través del cual pueden motivar a 
aprender a sus estudiantes? La pregunta es todavía más pertinaz, toda vez que la literatura sobre motivación escolar apunta a favorecer dinámicas que involucren al estudiante con el aprendizaje (HUERTAS, 1997; BORUCHOVITCH; BZUNECK; RUFINI, 2010; INGLÉS et al., 2014; LENS; MATOS; VANSTEENKISTE, 2015; MARTÍN; ROGRÍGUEZ, 2015; LIEURY; FENOUILLET, 2016).

Existe, tal vez, en estos estudiantes, una comprensión muy vinculada a la noción cotidiana de la motivación, donde el desarrollo disciplinar del constructo o no ha sido abordado durante la formación o no ha logrado superar la semantización que cotidianamente hacemos del concepto.

En lo anterior podría influir también el rol que tradicionalmente ha tenido el juego y lo lúdico, en especial en los primeros años de escolarización. Esto explicaría, en parte, que los profesores tiendan a utilizar el interés lúdico de los niños para diseñar actividades. Ciertamente esto es valioso considerando las ventajas en términos de desarrollo que presenta el juego como una experiencia de aprendizaje privilegiada (SARLÉ, 2001, 2008; WHITEBREAD et al., 2007). Sin embargo, pareciera que los futuros docentes centran su quehacer en los contenidos que deben manejarse en este nivel, utilizando el juego sólo como un detonador, dejando de lado su carácter de mediador del aprendizaje (GAUNTLETT et al., 2010). Lo cierto es que buen número de investigadores concuerda en el potencial educativo del juego, así como en la motivación por el aprendizaje que éstos pueden suscitar en los niños (GARVEY, 1977; ROSAS et al., 2003; JOHNSON; VILHJÁLMSSON; MARSELLA, 2005; GRAESSER et al., 2009; OLSON, 2010; CONNOLLY et al., 2012), especialmente en la perspectiva de aportar al niño el soporte cognitivo necesario para desarrollar procesos mentales de orden superior (VIGOTSKY, 1967; WHITEBREAD, 1997). Sin embargo, las declaraciones de los participantes no permiten visualizar la riqueza del juego como dispositivo de aprendizaje.

Claramente la entretención no lo es todo. Es un primer paso, pero debe estar orientado a suscitar el deseo de aprender. El problema, al parecer, es que las representaciones puestas en juego por los participantes remiten de alguna manera a una conceptualización más bien restringida de aprendizaje muy cercana a la idea de aprendizaje significativo de Ausubel (AUSUBEL; NOVACK; HEBESIAN, 1989; MOREIRA, 2012), sin poner en cuestión el hecho de que esta perspectiva teórica sólo da cuenta de un aprendizaje por recepción. En esta perspectiva, querer aprender no debiera significar sólo apropiarse de los contenidos escolares, sino que debiera ser comprendido como un proceso de apertura a la cultura y a una comprensión más rica del mundo gracias a la mediación de la escuela (ZAKHARTCHOUK, 1999; FALARDEAU; SIMARD, 2007).

No cabe duda que diseñar estrategias de motivación considerando los intereses lúdicos de los estudiantes puede ser una opción efectiva (SARLÉ, 2001), a condición de que los docentes incorporen el juego con la intencionalidad directa de generar aprendizajes. Sin embargo, la mayoría de los docentes en formación que respondieron con foco hacia la entretención entregó respuestas que no evidencian la intención clara de motivar hacia el aprendizaje por medio de las actividades lúdicas, más bien, se acota a propiciar una cierta atmósfera de agrado que haga posible la realización de la clase, resultados que son consistentes con lo hallado por Precht y otros autores (2016). 
Por otra parte, todas estas representaciones, ya sea en clave de entretener, realizar la tarea o de suscitar el deseo de aprender, contribuirían en su conjunto al logro de los aprendizajes esperados, a condición de que se aplicaran de modo integrado e intencionados. No obstante, muchos docentes en formación centran sus esfuerzos en diseñar actividades que entretengan al estudiante y no logran visualizar las otras dimensiones implicadas en el proceso.

Finalmente, dada la importancia que tiene la motivación escolar en el aprendizaje y las demandas explícitas al profesorado en orden a jugar un rol en la motivación de los estudiantes (CHILE, 2003; DÖRNYEI, 2008; DÖRNYEI; USHIODA, 2013; CHILE, 2014a, 2014b), las entidades formadoras de profesores deben hacerse responsables de la formación motivacional de los futuros docentes. Esto es particularmente importante en el caso chileno, toda vez que los resultados obtenidos sugieren que uno de cada dos docentes que egresa $(48,69 \%)$ no manejaría estrategias motivacionales focalizadas en el logro de aprendizajes, que permitan activar y estimular el deseo de querer aprender, restringiendo su horizonte a estrategias que apuntan a satisfacer más bien un requerimiento mínimo de seguridad y agrado para el estudiante. En consecuencia, probablemente el porcentaje de niños entretenidos en el aula será elevado, pero no sabemos cuántos de ellos querrán aprender los contenidos propuestos por la escuela.

\section{Referencias}

ALDERMAN, Kay. Motivation for achievement: possibilities for teaching and learning. New York: Routledge, 2013.

ALONSO TAPIA, Jesús. Motivación y aprendizaje en el ámbito de la compensación educativa e intercultural. Madrid: Facultad de Psicología, Universidad Autónoma de Madrid, 2009.

AMES, Carole. Classrooms: goals, structures, and student motivation. Journal of Educational Psychology, Washington, v. 84, n. 3, p. 261-271, 1992.

ANDERSON, Patricia. Cost perception and the expectancy-value model of achievement motivation. In: ANNUAL MEETING OF THE AMERICAN EDUCATIONAL RESEARCH ASOCIATION (AERA), 2000, New Orleans. Paper presented... New Orleans: AERA, 2000. p. 2-6. Trabajo presentado en creating knowledge in the 21st century: insights from multiple perspectives. Disponivel em: <https://files.eric.ed.gov/fulltext/ED441020. pdf >. Acesso em: 10/03/2017.

AUSUBEL, David; NOVACK, Joseph; HENESIAN, Helen. Psicología educativa: un punto de vista cognoscitivo. México, DF: Trillas, 1989.

BANDURA, Albert; LOCKE, Edwin. Negative self-efficacy and goal effects revisited. Journal of Applied Psychology, Washington, DC, v. 88, n. 1, p. 87-99, 2003.

BANDURA, Albert; RAMACHAUDRAN, Vilayanur. Self-efficacy. In: RAMACHAUDRAN, Vilayanur (Ed.). Encyclopedia of human behavior. New York: Academic Press, 1994. p.71-81. 
BARRON, Kenneth E.; HULLEMAN, Chris S. Expectancy-value-cost model of motivation. In: WRIGHT, James D. (Ed.). International encyclopedia of social and behavioral sciences. Oxford: Elsevier, 2015. p .261-271.

BONG, Mimi. Role of self-efficacy and task-value in predicting college students' course performance and future enrollment intentions. Contemporary Educational Psychology, Amsterdam, v. 26, n. 4, p. 553-570, 2001.

BORUCHOVITCH, Evely; BZUNECK, José Aloyseo. A motivação do aluno. Petrópolis: Vozes, 2000.

BORUCHOVITCH, Evely; BZUNECK, José Aloyseo; RUFINI, Sueli. E. Motivação para aprender. Petrópolis: Vozes, 2010.

CERASOLI, Christopher; NICKLIN, Jessica; FORD, Michael. Intrinsic motivation and extrinsic incentives jointly predict performance: A 40-year meta-analysis. Psychological Bulletin, Washington, v. 140, n. 4, p. 980-1080, 2014.

CHILE. Ministerio de Educación. Fundamentos, otros indicadores de calidad educativa: unidad de currículum y evaluación. Santiago de Chile: Ministerio de Educación, 2014a.

CHILE. Ministerio de Educación. Marco para la buena enseñanza: Centro de Perfeccionamiento, Experimentación e Investigaciones Pedagógicas (CPEIP). Santiago de Chile: Ministerio de Educación, 2003.

CHILE. Ministerio de Educación. Otros indicadores de calidad educativa. Santiago de Chile: Ministerio de Educación, 2014b.

CONNOLLY, Thomas et al. A systematic literature review of empirical evidence on computer games and serious games. Computers \& Education, Amsterdam, v. 59, n. 2, p. 661-686, 2012.

CONTRERAS-SANZANA, Gladys; VILLALOBOS-CLAVERÍA, Alejandro. La formación de profesores en Chile: una mirada a la profesionalización docente. Educación y Educadores, Bogotá, v. 13, n. 3, p. 397-417, 2010.

DÖRNYEI, Zoltán. Estrategias de motivación en el aula de lenguas. Barcelona: UOC, 2008.

DÖRNYEI, Zoltán; USHIODA, Ema. Teaching and researching: motivation. Horlow: Pearson, 2013.

ECCLES, Jacquelynne. Subjective task value and the Eccles et al. model of achievement-related choices. In: ELLIOT, Andrew; DWECK, Carol (Ed.). Handbook of competence and motivation. New York: Guilford, 2005. p. 105-121.

ELLIOT, Andrew et al. Achievement goals, performance contingencies, and performance attainment: an experimental test. Journal of Educational Psychology, Washington, DC, v. 97, n. 4, p. 630-640, 2005.

FALARDEAU, Erick; SIMARD, Denis. Rapport à la culture et approche culturelle de l'enseignement. Canadian Journal of Education / Revue Canadienne de L'éducation, Ottawa, v. 30, n. 1, p. 1-24, 2007.

FLAKE, Jessica Kay et al. Measuring cost: the forgotten component of expectancy-value theory. Contemporary Educational Psychology, Amsterdam, v. 41, p. 232-244, 2015. 
GARCÍA LÓPEZ, Juan. La "Need achievemente" teoría clásica para la explicación de la motivaciñon para el rendimiento escolar. Ensayos, Albacete, v. 19, p. 345-364, 2008.

GARVEY, Catherine. El juego infantil. Madrid: Morata, 1977.

GAUNTLETT, David et al. The future of play. Billund: Lego, 2010.

GIBBS, Graham. El análisis de datos en investigación cualitativa. Madrid: Morata, 2012.

GOLLWITZER, Peter; OETTINGEN, Gabriele. Motivation: the history of a concept. In: SMELSER, Neil J.; BALTES, Paul B. (Ed.). International encyclopedia of the social and behavioral sciences. Oxford: Elsevier, 2001. p. 10109-10112.

GORICHON, Solange et al. Relaciones entre formación inicial e iniciación profesional de los docentes: principios y desafíos para la formación práctica. Santiago de Chile: Universidad Alberto Hurtado, 2015. (Cuadernos de educación).

GRAESSER, Arthur et al. Deep learning and emotion in serious games. In: RITTERFELD, Ute et al. (Ed.). Serious games: mechanism and effects. London: Routledge, 2009. p. 83-102.

HATTIE, John. Visible learning: a synthesis of 800+ meta-analyses on achievement. Oxford: Routledge, 2009.

HUERTAS, José Antonio. Motivación: querer aprender. Buenos Aires: Aique, 1997.

IGLESIA, María Lina. Observación y evaluación del ambiente de aprendizaje en educación infantil: dimensiones y variables a considerar. Revista Iberoamericana de Educación, Madrid, v. 47, p. 49-70, 2008.

INGLÉS, Cándido et al. Perfiles de orientaciones de metas y autoconcepto de estudiantes de educación secundaria. Revista de Psicodidáctica, Amsterdam, v. 20, n. 1, p. 99-116, 2014.

JOHNSON, Lewis; VILHJÁLMSSON, Hannes Högni; MARSELLA, Stacy. Serious games for language learning: how much game, how much Al? In: LOOI, Chee-Kit et al. Artificial Intelligence in Education: supporting learning through intelligent and socially informed technology. Amsterdam: IOS Press, 2005. p. 306-313.

JUVONEN, Jaana; WENTZEL, Kathryn. Social motivation: understanding children's school adjustment. Cambridge: Cambridge University Press, 1996.

KIM, Tae-Young; KIM, Yoon-Kyoung. A quasi-longitudinal study on English learning motivation and attitudes: the case of South Korean students. The Journal of AsiaTEFL, Seul, v. 13, n. 2, p. 138-155, 2016.

LENS, Willy; MATOS, Lennia; VANSTEENKISTE, Maarten. El profesor como fuente de motivación de los estudiantes: hablando del qué y del por qué del aprendizaje de los estudiantes. RIDU Revista Digital de Investigación en Docencia Universitaria, Lima, v. 4, n. 1, p. 1-9, 2015.

LIEURY, Alain; FENOUILLET, Fabien. Motivación y éxito escolar. México, DF: Fondo de Cultura Económica, 2016. 
LÓPEZ, Verónica; BILBAO, María de los Ángeles; RODRIGUEZ, Juan Ignacio. La sala de clases sí importa: incidencia del clima de aula sobre la percepción de intimidación y victimización entre escolares. Universitas Psychologica, Bogotá, v. 11, n. 1, p. 91, 2012.

MALLART NAVARRA, Joan. Didáctica: del currículum a las estrategias de aprendizaje. Revista Española de Pedagogía, Madrid, v. 58, n. 217, p. 417-438, 2000.

MARTín, Alba; ROGRíGUEZ, Susana. Motivación en alumnos de Primaria en aulas con metodología basada en proyectos. Revista de Estudios e Investigación en Psicología y Educación, A Coruña, v. extra, n. 1, p. 58-62, 2015.

MARZANO, Robert et al. Dimensions of learning. México, DF: Iteso, 2005.

MORA, Angela; CRUZ, Jaume; SOUSA, Catharina. How to improve the motivational climate and communication styles in the field of physical education and sport. Infancia y Aprendizaje, London, v. 36, n. 1, p. 91-103, 2013.

MOREIRA, Marco Antônio. Aprendizaje significativo, campos conceptuales y pedagogía de la autonomía: implicaciones para la enseñanza. Aprendizagem Significativa em Revista / Meaningful Learning Review, Porto Alegre, v. 2, n. 1, p. 44-65, 2012.

NEUVILLE, Sandrine; BOURGEOIS, Étienne; FRENAY, Mariane. The subjective task value: clarification of a construct. In: NEUVILLE, Sandrine (Ed.). La perception de la valeur des activités d'apprentissage: étude des déterminants et effets. 2004. 248 p. (Tesis doctoral) - Faculté de Psychologie et des Sciences de l'Éducation. Université Catholique de Louvain, Louvain la Neuve, 2004. p. 111-137.

OLSON, Cheryl. Children's motivations for video game play in the context of normal development. Review of General Psychology, Washington, DC, v. 14, n. 2, p. 180-187, 2010.

PINTRICH, Paul. The role of goal orientation in self-regulated learning. In: BOEKAERTS, Monique et al. (Ed.). Handbook of self regulation. San Diego: Academic Press, 2000. p. 451-501.

PRECHT, Andrea et al. Familia y motivación escolar: desafíos para la formación inicial docente. Estudios Pedagógicos, Valdivia, v. 42, n. 4, p. 165-182, 2016.

PREISS, David. The Chilean instructional pattern for the teaching of language: a video-survey study based on a national program for the assessment of teaching. Learning and Individual Differences, Amsterdam, v. 19, n. 1, p. 1-11, 2009.

ROBBINS, Steven et al. Do psychosocial and study skill factors predict college outcomes? A meta-analysis. Psychological Bulletin, Washington, DC, v. 130, n. 2, p. 261-288, 2004.

ROSAS, Ricardo et al. Beyond nintendo: design and assessment of educational video games for first and second grade students. Computers \& Education, Amsterdam, v. 40, n. 1, p. 71-94, 2003. 
RYAN, Richard; DECl, Edward. Self-determination theory: basic psychological needs in motivation, development, and eellness. New York: Guilford Press, 2017.

SARLÉ, Patricia Mónica. Enseñar en clave de juego. Buenos Aires: Novedades Educativas, 2008.

SARLÉ, Patricia Mónica. Juego y aprendizaje escolar: los rasgos del juego en la educación infantil. Buenos Aires: Novedades Educativas, 2001.

SILVA-PEÑA, Ilich; NAVARRETE, Pamela; ZÚÑIGA, María José. Motivación escolar: la percepción de profesores en ejercicio y estudiantes de pedagogía. Temuco: Universidad Católica de Temuco, 2012. (Informe Grupo Focal Fondecyt; 1120351).

SIMÓN, Cecilia; GÓMEZ, Pilar; ALONSO-TAPIA, Jesús. Prevención de la disrupción en el aula: papel del clima motivacional de clase y de las estrategias de afrontamiento. Cultura y Educación, Oxfordshire, v. 25, n. 1, p. 49-64, 2013.

THOONEN, Erick et al. Can teachers motivate students to learn? Educational Studies, Oxfordshire, v. 37, n. 3, p. 345-360, 2011.

VALENZUELA, Jorge. Características psicométricas de una escala para caracterizar el sentido del aprendizaje escolar. Universitas Psychologica, Bogotá, v. 8, n. 1, p. 49-59, 2009.

VALENZUELA, Jorge. Más allá de la tarea: pistas para una redefinición del concepto de motivación escolar. Educação e Pesquisa, São Paulo, v. 33, n. 3, p. 409-426, 2007.

VALENZUELA, Jorge et al. Estudio de las representaciones de la motivación escolar, autoeficacia y sentido del aprendizaje escolar: aportes para el mejoramiento de la formación motivacional de los docentes. Santiago de Chile: Fondo Nacional de Ciencia y Tecnología, 2014. (Informe final fondecyt regular; 1120351).

VALENZUELA, Jorge et al. Característica psicométricas de un inventario para explorar la autoeficacia motivacional docente (IAM-D). Revista Mexicana de Investigación Educativa, México, DF, v. 20, n. 66, p. 859-878, 2015.

VIAU, Roland. La motivation en contexte scolaire. Bruxelles: De Boeck, 2009.

VIGOTSKY, Lev Seminovich. Play and its role in the mental development of the child. Soviet Psychology, v. 5, p. 6-18, 1967.

WHITEBREAD, David. Developing children's problem-solving: the educational uses of adventure games. In: McFARLANE, Angela (Ed.). Information technology and authentic learning. London: Routledge, 1997. p. 13-39.

WHITEBREAD, David et al. Development of metacognition and self-regulated learning in young children: role of collaborative and peer-assisted learning. Journal of Cognitive Education and Psychology, New York, v. 6, n. 3, p. 433-455, 2007. 
WIGFIELD, Allan; CAMBRIA, Jenna. Expectancy-value theory: retrospective and prospective. In: URDAN, Timothy C.; KARABENICK, StuartA. (Ed.). Advances in motivation and achievement. v. 16A. Bingley: Emerald, 2010. p. 35-70.

WIGFIELD, Allan; TONKS, Stephen; KLAUDA, Susan. Expectancy-value theory. In: WENTZEL, Kathryn R.; MIELE, David B. (Ed.). Handbook of motivation at school. London: Routledge, 2009. p. 55-75.

ZAKHARTCHOUK, Jean.-Michel. L'enseignant, un passeur culturel. Paris: ESF, 1999.

Recibido en: 02.06.2017 Modificaciones en: 25.10.2017 Aprobado en: 29.11.2017

Jorge Valenzuela es doctor en Educación e investigador en el Centro de Estudios Avanzados de la Universidad de Playa Ancha.

Carla Muñoz es doctora en Psicología y Ciencias de la Educación y se desempeña como académico en la Escuela de Pedagogía de la Pontificia Universidad Católica de Valparaíso.

Abigail Montoya Levinao es profesora de Educación básica e investigadora del Equipo de Investigación en Creencias, Motivación y Aprendizaje.

\section{ERRATA}

Fe de erratas: http://dx.doi.org/10.1590/s1678-4634201844179652E

En el artículo "Estrategias motivacionales efectivas en profesores en formación", número de DOl: http://dx.doi.org/10.1590/s1678-4634201844179652, publicado en el periódico Educação e Pesquisa, Volumen 44, 2018.

En el nombre del tercer autor, los dos apellidos deben ser destacados y considerados en la indexación, Donde se leía:

"Abigail Montoya Levinao"

Debe leerse:

"Abigail MONTOYA LEVINAO" 